\title{
Fuzzy Ants and Clustering
}

\author{
Parag M. Kanade and Lawrence O. Hall \\ Dept. of Computer Science and Engineering, ENB118 \\ University of South Florida \\ Tampa, Fl. 33620 \\ hall@csee.usf.edu
}

March 14, 2006

\begin{abstract}
A Swarm Intelligence inspired approach to clustering data is described. The algorithm consists of two stages. In the first stage of the algorithm ants move the cluster centers in feature space. The cluster centers found by the ants are evaluated using a reformulated fuzzy $\mathrm{C}$ Means criterion. In the second stage the best cluster centers found are used as the initial cluster centers for the fuzzy C Means algorithm. Results on 18 data sets show that the partitions found using the ant initialization are better optimized than those obtained from random initializations. The use of a reformulated fuzzy partition validity metric as the optimization criterion is shown to enable determination of the number of cluster centers in the data for several data sets. Hard $\mathrm{C}$ Means was also used after reformulation and the partitions obtained from the ant based algorithm were better optimized than those from randomly initialized hard $\mathrm{C}$ Means.
\end{abstract}

Keywords: Clustering, Swarm Intelligence, Ant Colony Optimization, Fuzzy C Means, Hard C Means, fuzzy partition validity

\section{Introduction}

Modern technology provides us with efficient and low-cost techniques for data collection. Raw data, however, is of limited use for decision making and intelligent analysis. Machine learning aims to create automatic or semiautomatic tools for the analysis of raw data to discover useful patterns and rules. Clustering is one of the most important unsupervised learning techniques $[1,2]$.

Clustering approaches are typically quite sensitive to initialization. In this paper, we examine a swarm inspired approach to building clusters which allows for a more global search for the best partition than iterative optimization approaches. The approach is described with 
cooperating ants as its basis. The ants participate in placing cluster centroids in feature space. They produce a partition which can be utilized as is or further optimized. The further optimization can be done via a focused iterative optimization algorithm.

Experiments were done with both deterministic algorithms which assign each example to one and only one cluster and fuzzy algorithms which partially assign examples to multiple clusters. The algorithms are from the C-means family [3]. These algorithms were integrated with swarm intelligence concepts to result in clustering approaches that were less sensitive to initialization. The clustering approach introduced here provides a framework for optimization of most any objective function that can be expressed in terms of cluster centroids. It is highly parallelizable which could enable the time cost to be the same or lower than classical clustering. The algorithm provides a high likelihood of skipping most poor local solutions resulting in a quality partition of data. The new algorithms are loosely based on cemetery organization and brood sorting as done by ants [4].

The algorithm introduced here requires the number of clusters be known, but has minimal sensitivity to parameter choices and results in clusters which are often better optimized than those from current algorithms. Further, we show that it can be integrated with a cluster validity metric to potentially discover the number of classes in the data.

The paper proceeds in Section 2 with a discussion of swarm intelligence, the clustering algorithms used and related work. Section 3 contains a description of applying the ants to centroids of clusters. Section 4 discusses the data sets used in evaluating the performance and Section 5 contains the results of applying the centroid based algorithm utilizing fuzzy clustering and Section 6 utilizing hard clustering, Section 7 explores execution time, Section 8 is a discussion including discovering the number of clusters and Section 9 contains conclusions.

\section{Swarm Intelligence and Clustering}

Research in using the social insect metaphor for solving problems is still in its infancy. The systems developed using swarm intelligence principles emphasize distributiveness, direct or

indirect interactions among relatively simple agents, flexibility and robustness [4]. Successful applications have been developed in the communication networks, robotics and combinatorial optimization fields. 


\subsection{Cemetery Organization and Brood Sorting in Ants}

Many species of ants cluster dead bodies to form cemeteries, and sort the larvae into several piles [4]. This behavior can be simulated using a simple model in which the agents move randomly in space and pick up and deposit items on the basis of local information. The clustering and sorting behavior of ants can be used as a metaphor for designing new algorithms for data analysis and graph partitioning. The objects can be considered as items to be sorted. Objects placed next to each other have similar attributes. This sorting takes place in two-dimensional space, offering a low-dimensional representation of the objects.

Most swarm clustering work has followed the above model. In our work, there is implicit communication among the ants making up a partition. The ants also have memory. However, they do not pick up and put down objects but rather place summary objects in locations and remember the locations that are evaluated as having good objective function values. The objects represent single dimensions of multidimensional cluster centroids which make up a data partition.

\subsection{Clustering}

The aim of cluster analysis is to find groupings or structures within unlabeled data [5]. The partitions found should result in similar data being assigned to the same cluster and dissimilar data assigned to different clusters.

In most cases the data is in the form of real-valued vectors. The Euclidean distance is one measure of similarity for these data sets.

Clustering techniques can be broadly classified into a number of categories [6]. In this paper algorithms from the following categories are used:

- Deterministic crisp: Each example is assigned to one and only one cluster.

- Possibilistic/Fuzzy: Degrees of membership indicate the extent to which the example belongs to the cluster. The sum of memberships of each example across all the clusters may not be 1 for possibilistic clustering, but is equal to 1 in the fuzzy case.

\subsubsection{Hard Clustering}

Hard C Means (HCM) is one of the simplest unsupervised clustering algorithms for a fixed number of clusters. The basic idea of the algorithm is to initially guess the centroids of the clusters and then refine them. Cluster initialization is very crucial because the algorithm is very sensitive to this initialization. A good choice for the initial cluster centers is to place them as far away from each other as possible. The nearest neighbor algorithm is then used 
to assign each example to a cluster. Using the clusters obtained, new cluster centroids are calculated. The above steps are repeated until there is no significant change in the centroids.

The objective function minimized by the hard C Means algorithm is given in (1).

$$
J=\sum_{i=1}^{c} \sum_{k=1}^{n} D_{i k}\left(x_{k}, \beta_{i}\right)
$$

where

$c \geq 2:$ Number of clusters

$n$ : Number of data points

$\beta_{i}$ : The $i^{\text {th }}$ cluster prototype and $x_{k}$ the kth data vector

$D_{i k}\left(x_{k}, \beta_{i}\right)$ : Distance of $x_{k}$ from $i^{\text {th }}$ cluster center

\subsubsection{Fuzzy Clustering}

Hard clustering algorithms assign each example to one and only one cluster. This model is inappropriate for real data sets in which the boundaries between the clusters may not be well defined. Fuzzy algorithms can partially assign data to multiple clusters. The strength of membership in the cluster depends on the closeness of the example to the cluster center.

The Fuzzy C Means algorithm (FCM) [7], allows an example to be a partial member of more than one cluster. The FCM algorithm is based on minimizing the objective function (2) with the algorithm shown in Figure 1.

$$
J_{m}(U, \beta)=\sum_{i=1}^{c} \sum_{k=1}^{n} u_{i k}^{m} D_{i k}\left(x_{k}, \beta_{i}\right)
$$

where

$u_{i k}:$ Membership of the $k^{\text {th }}$ object in the $i^{\text {th }}$ cluster

$\beta_{i}$ : The $i^{\text {th }}$ cluster prototype

$m \geq 1$ : The degree of fuzzification

$c \geq 2:$ Number of clusters

$n:$ Number of data points

$D_{i k}\left(x_{k}, \beta_{i}\right):$ Distance of $x_{k}$ from $i^{\text {th }}$ cluster center

The drawback of clustering algorithms like FCM and HCM, which are based on the hill climbing heuristic is, prior knowledge of the number of clusters in the data is required and they have significant sensitivity to cluster center initialization [8].

\subsubsection{Work on Ant based clustering}

There has been work on clustering data utilizing swarm intelligence and we describe the most related work here. A number of approaches $[9,10,11,12,13,14]$ project the data to be clustered onto a grid based on the approach from [15]. In [10] a small number of web pages 
1. Initialize the initial cluster centers $\beta^{0}$

2. Update the membership matrix $\mathrm{U}, U^{t}, U^{(t-1)}$

$$
u_{i k}=\frac{1}{\sum_{j=1}^{c}\left(\frac{D_{i k}\left(x_{k}, \beta_{i}\right)}{D_{i j}\left(x_{k}, \beta_{j}\right)}\right)^{\frac{1}{1-m}}}
$$

3. At the $t^{\text {th }}$ step, calculate the new cluster centers

$$
\beta_{i}=\frac{\sum_{k=1}^{n} u_{i k}^{m} \cdot x_{k}}{\sum_{k=1}^{n} u_{i k}^{m}}
$$

4. If $\left|\beta^{t}-\beta^{t-1}\right|<\epsilon$ then STOP; otherwise goto step 2

Figure 1: Fuzzy C Means Algorithm

from four classes were clustered into groups that were reasonably homogeneous. Promising results on artificial data were obtained by allowing ants to have short-term memory in traversing a portable grid to group documents in [13]. In [16, 17] some improvements have been made to the algorithms for utilizing ants to perform clustering on a grid. Results are compared with K-means, as done here. Mostly synthetic data sets were used with three real data sets. They utilized the F-measure which requires class labels and sometimes obtained partitions better than K-means, especially when there are unequal size clusters. They argue that their approach is pretty good for picking the number of cluster centers (which K-means cannot do). They get results that are pretty consistently close to or better than K-means with the correct number of clusters. They utilized the cosine distance, while we used the Euclidean distance.

In a gray level image segmentation problem domain [18], an interesting approach was proposed where an ant produces a partition by making a single pass through the data and assigning data to cluster centers. Multiple ants (10) do this and pheromone trails are used to guide the assignment of objects to classes. Only the ant that produces the best partition is allowed to cause an update to the pheromone trails. This is a bit of an ensemble clustering approach [19] and shows some clear promise with a noisy image.

In [20] the ants were used to create a hierarchical cluster partition. There is one ant for every object and they aligned themselves into a tree structure. The F-measure is used to evaluate the partitions and to show that some partitions are better than what you will get with $\mathrm{K}$-means which is not hierarchical.

In [21] Ants group feature vectors using the concept of odor. Feature vectors with a similar odor are assigned to the same cluster. Each ant is given a feature vector and a class label (initially none). They cannot change objects, but they can change class depending 
upon the class of other objects to which they have a similar odor (as determined by a similarity metric). Since clusters or classes can be created, this algorithm can automatically find the number of clusters. Experimental results are shown on 13 data sets ( 8 artificial) and compared to K-means which is always initialized with 10 clusters. Usually, the F-measure (which requires class labels) shows this approach to be better. However, K-means might more typically be initialized with some number of clusters closer to the true number.

\section{$3 \quad$ Fuzzy ant clustering with centroids}

The ant based clustering algorithms discussed so far cluster data by moving the objects in a $2 \mathrm{D}$ space and merging them to form clusters. Another avenue we have pursued is to allow the ants to relocate cluster centroids in feature space. The formulation is similar at a very high-level to what was done in [22], but ants are utilized rather than a genetic approach.

In the algorithm discussed here, the stochastic property of ants was simulated to obtain good cluster centers. The ants move randomly in the feature space carrying a feature of a cluster center with them. After a fixed number of iterations the cluster centers are evaluated using the reformulation of FCM which leaves out the membership matrix [23]. After the ant stage the best cluster centers obtained are used as the initial cluster centers for the FCM and HCM algorithms.

The approach presented here does a type of global, cooperative, directed search for the optimal cluster centroids. Groups of ants cooperate in finding the optimal centroid values for the optimal partition. It might be compared with Fuzzy J-means [24] which uses directed local neighborhood search in an attempt to find a global minima or at least skip local extrema. They remove a centroid and replace it with an unoccupied pattern which is far from existing centroids (though how exactly a pattern is chosen is not defined), they keep doing this with some iterations of the algorithm to get new partitions. They show better optimizations are obtained when the number of clusters is much greater than the true number. There is not much difference in performance when they are close to the true number of clusters which is of interest here.

We explore how our algorithm performs if it is viewed as an initialization algorithm. In this framework, it can be compared to [25] where one-dimensional clustering was used to find initializations. They report error on data sets, which is not necessarily the same as the best optimized partition for which we search here. They do not compare their approach with the best result from a random set of initializations done in the same time it takes to produce a partition in their approach. In [26], four different initialization methods for K-means were explored. It was found that, on average, random initialization was better than all but one method and could not be shown to be statistically inferior to the "Kaufman" initialization. 


\subsection{Reformulation of Clustering Criteria for FCM and HCM}

In [23] the authors have proposed a reformulation of the optimization criteria used in a couple of common clustering objective functions. The original clustering functions minimize the objective function (3) to find good clusters.

$$
J_{m}(U, \beta)=\sum_{i=1}^{c} \sum_{k=1}^{n} U_{i k}^{m} D_{i k}\left(x_{k}, \beta_{i}\right)
$$

where

$U_{i k}:$ Membership of the $k^{\text {th }}$ object in the $i^{\text {th }}$ cluster

$\beta_{i}$ : The $i^{\text {th }}$ cluster prototype

$m \geq 1$ : The degree of fuzzification

$c \geq 2:$ Number of clusters

$n:$ Number of data points

$D_{i k}\left(x_{k}, \beta_{i}\right)$ : Distance of $x_{k}$ from $i^{\text {th }}$ cluster center

The reformulation replaces the membership matrix $U$ with the necessary conditions which are satisfied by $U$. The reformulated version of $J_{m}$ is denoted as $R_{m}$.

For the Hard clustering case the $U$ optimization is over a crisp membership matrix. The necessary condition for $U$ is given in Equation 4. Equation 5 gives the necessary conditions for $U$, for the fuzzy case. The distance $D_{i k}\left(x_{k}, \beta_{i}\right)$ is denoted as $D_{i k}$.

$$
\begin{aligned}
U_{i k} & =0 \text { if } D_{i k}>\min \left(D_{1 k}, D_{2 k}, D_{3 k}, \cdots, D_{c k}\right) \\
& =1 \text { otherwise }
\end{aligned}
$$

$$
U_{i k}=\frac{\left(D_{i k}^{\frac{1}{1-m}}\right)}{\left(\sum_{j=1}^{c} D_{j k}^{\frac{1}{1-m}}\right)}
$$

The reformulations for hard and fuzzy optimization functions are given in equations 6 and 7 respectively. The function $R$ depends only on the cluster prototype and not on the $U$ matrix, whereas $J$ depends on both the cluster prototype and the $U$ matrix. The $U$ matrix for the reformulated criterion can be easily computed using Equation 4 or 5.

$$
\begin{gathered}
R_{1}(\beta)=\sum_{k=1}^{n} \min \left(D_{1 k}, D_{2 k}, \cdots, D_{c k}\right) \\
R_{m}(\beta)=\sum_{k=1}^{n}\left(\sum_{i=1}^{c} D_{i k}^{\frac{1}{1-m}}\right)^{1-m}
\end{gathered}
$$




\subsection{Algorithm}

A partition of data can be compactly described by a set of $c$ cluster centroids in feature space. Clustering algorithms which produce centroids attempt to position the centroids, in feature space, in such a way as to minimize (or maximize) an objective function. Each centroid is described by $s$ feature values. If an ant is assigned to move a feature value, in a normalized feature space, it is helping search for an extrema of the objective function. For a given partition, a unique ant is assigned to a given feature of a given cluster. There will then be $c \times s$ ants involved in determining the structure of a partition. They will position the features of the centroids, thereby positioning the centroids, and creating a data partition (each example belongs partially or fully to the nearest cluster(s)). Each ant has a memory of the $\mathrm{b}=5$ most optimal locations visited. When the ant stops and the current partition is evaluated, it will appropriately replace the least good rank order stored position when the current position results in a more optimal partition.

The evaluation is done through an objective function for the particular cluster based algorithm to be optimized. This forms the basis for the clustering algorithm presented here. The group of ants cooperate to find the best partition, but work independently (though often from locations remembered as being good) to find a new location which will result in a new data partition.

The ants co-ordinate to move cluster centers in feature space in the search for optimal cluster centers. Initially the feature values are normalized between 0 and 1 . Each ant is assigned to a particular feature of a cluster in a partition. The ants never change the feature, cluster or partition assigned to them. A pictorial view is given in Figure 2 where each vertical line is a dimension in parallel coordinates $[27,28]$. The links between ants show that a group of four are taken as working together. For, example they would represent the location of a cluster in a 4D feature space. After randomly moving the cluster centers for a fixed number of iterations, called an epoch, the quality of the partition is evaluated by using the reformulated criterion 6 or 7 . If the current partition is better than any of the previous partitions in the ant's memory then the ant remembers this partition else the ant, with a given probability goes back to a better partition or continues from the current partition. This ensures that the ants do not remember a bad partition and erase a previously known good partition. Even if the ants change good cluster centers to unreasonable cluster centers, the ants can go back to the good cluster centers as the ants have a finite memory in which they keep the best of the visited cluster center locations. There are two directions for the random movement of the ant. The positive direction is when the ant is moving in the feature space from 0 to 1 , and the negative direction is when the ant is moving in the feature space 
from 1 to 0 . If during the random movement the ant reaches the end of the feature space the ant reverses direction. After a fixed number of epochs the ants stop.

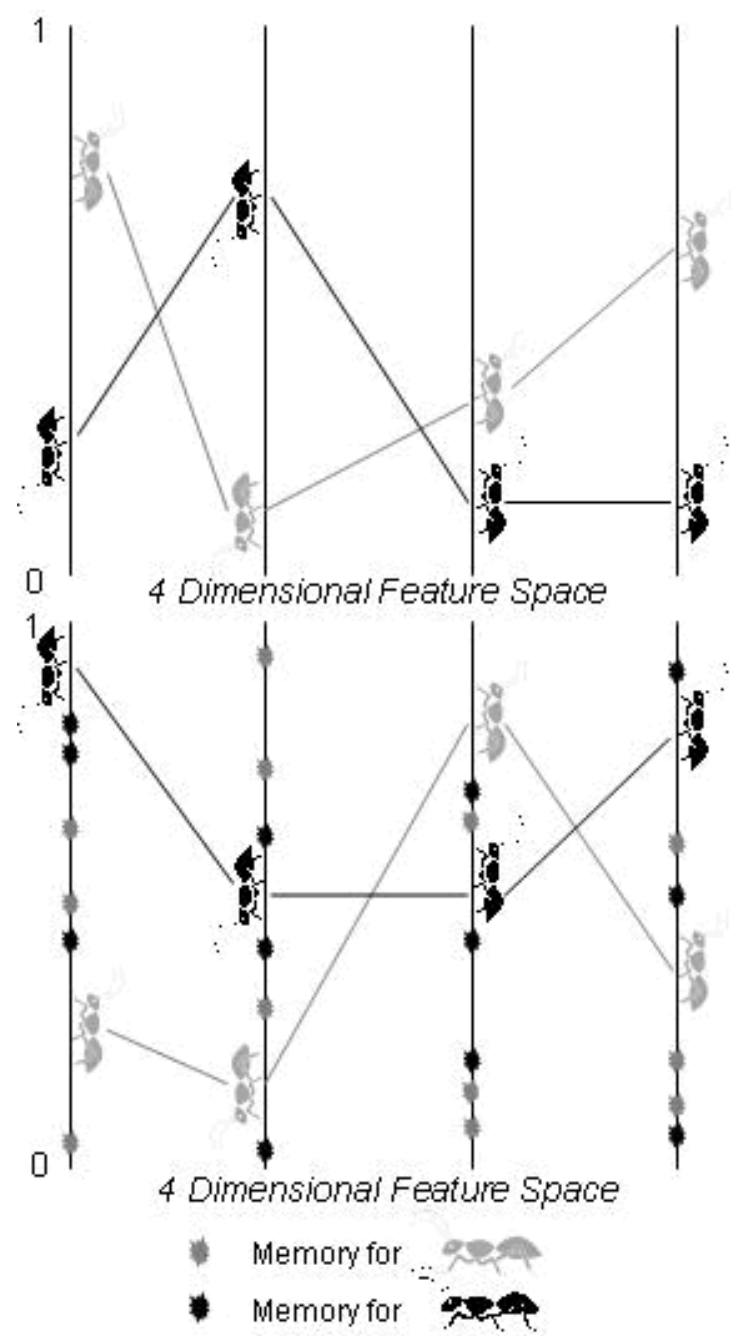

Figure 2: Pictorial view of the algorithm

The data is partitioned using the centroids obtained from the best known $R_{m}$ value. The nearest neighbor algorithm is used for assignment to a cluster. The cluster centers so obtained are then used as the initial cluster centers for the FCM or the HCM algorithm. The ant based algorithm is presented in Figure 3.

The values of the parameters used in the algorithm are shown in Table 1.

\section{Data Sets}

Six real data sets and ten artificial data sets were used in the experiments. The data sets were: the Iris Plant Data Set, Wine Recognition Data Set, Glass Identification Data Set, 
1. Normalize the feature values between 0 and 1 . The normalization is linear. The minimum value of a particular feature is mapped to 0 and the maximum value of the feature is mapped to 1 .

2. Initialize the ants with random initial values and with random direction. There are two directions, positive and negative. The positive direction means the ant is moving in the feature space from 0 to 1 . The negative direction means the ant is moving in the feature space from 1 to 0 . Clear the initial memory. The ants are initially assigned to a particular feature within a particular cluster of a particular partition. The ants never change the feature, cluster or the partition assigned to them.

3. Repeat

3.1 For one epoch /* One epoch is $n$ iterations of random ant movement */

\subsubsection{For all ants}

3.1.1.1 With a probability $P_{\text {rest }}$ the ant rests for this epoch

3.1.1.2 If the ant is not resting then with a probability $P_{\text {continue }}$ the ant continues in the same direction else it changes direction

3.1.1.3 With a value between $D_{\min }$ and $D_{\max }$ the ant moves in the selected direction

3.2 The new $R_{m}$ value is calculated using the new cluster centers calculated by recording the position of the ants known to move the features of clusters for a given partition

3.2.1 If the partition is better than any of the old partitions in memory then the worst partition is removed from the memory and this new partition is copied to the memories of the ants making up the partition

3.2.2 If the partition is not better than any of the old partitions in memory Then

With a probability $P_{\text {ContinueCurrent }}$ the ant continues with the current partition Else

With a probability 0.6 the ant moves to the best known partition, with a probability 0.2 the ant moves to the second best known partition, with a probability 0.1 the ant goes to the third best known partition, with a probability 0.075 the ant goes to the fourth best known partition and with a probability 0.025 the ant goes to the worst known partition

Until Stopping criteria

The stopping criterion is the number of epochs.

Figure 3: Fuzzy ant clustering with centroids algorithm 
Table 1: Parameter Values. Note the multiplier 30 for the number of ants allows for 30 partitions.

\begin{tabular}{|l|r|}
\hline \multicolumn{1}{|c|}{ Parameter } & \multicolumn{2}{c|}{ Value } \\
\hline Number of ants & $30 \times c \times$ \#features \\
\hline Memory per ant & 5 \\
\hline Iterations per epoch & 50 \\
\hline Epochs & 1000 \\
\hline$P_{\text {rest }}$ & 0.01 \\
\hline$P_{\text {continue }}$ & 0.75 \\
\hline$P_{\text {ContinueCurrent }}$ & 0.20 \\
\hline$D_{\text {min }}$ & 0.001 \\
\hline$D_{\text {max }}$ & 0.01 \\
\hline
\end{tabular}

Multiple Sclerosis Data Set, MRI Data Set, British Towns'Data Set, Gauss 1-5 Data Sets, Gauss500 1-5 Data Sets. They are described in Table 2.

Table 2: Data Sets

\begin{tabular}{|l|r|r|r|}
\hline Data Set & \# Examples & $\begin{array}{c}\text { \# Continuous } \\
\text { Attributes }\end{array}$ & \# Classes \\
\hline Iris & 150 & 4 & 3 \\
\hline Wine & 178 & 13 & 3 \\
\hline Glass & 214 & 9 & 6 \\
\hline MRI & 65536 & 3 & 3 \\
\hline Multiple Sclerosis & 98 & 5 & 2 \\
\hline British Towns & 50 & 4 & 5 \\
\hline Gauss1-5 & 1000 & 2 & 5 \\
\hline Gauss500-1-5 & 500 & 2 & 5 \\
\hline
\end{tabular}

Ten artificial data sets were generated from a mixture of five Gaussians. The probability distribution across all the data sets is the same but the means and standard deviations of the Gaussians are different. Of the ten data sets, five data sets had 500 instances each and the remaining five data sets had 1000 instances each. Each instance had two attributes. The parameters used to generate the data sets are shown in Appendix A.

To visualize the Iris data set, the Principal Component Analysis (PCA) algorithm [29] was used to project the data points into a 2D and 3D space. Figure 4 shows the scatter 
of the points, after PCA, in 2D for the Iris data set. One class is linearly separable from the other two. For clustering purposes, the Iris data set can be considered as having only 2 clusters. The age factor plays an important role in the Multiple Sclerosis data set. For this data set we perform the experiments twice, once considering the age feature and once ignoring the age feature.

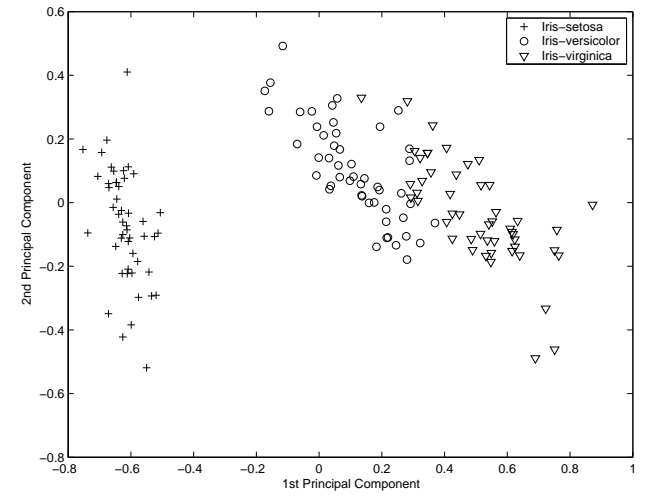

Figure 4: Iris Data Set (Normalized)- First 2 Principal Components

\section{Results for Fuzzy ant clustering with centroids algo- rithm}

The algorithm of Figure 3 was applied to the six real data sets and ten artificial data sets described earlier.

The results obtained for the data sets are shown in Table 3. The results for the FCM and $\mathrm{HCM}$ are the average results from 50 random initializations. The glass data set has been simplified to have just 2 classes window glass and non-window glass. The results for this modified data set are also shown in Table 3. The attribute age plays an important role in the Multiple Sclerosis data set; the results considering the age feature and ignoring the age feature are also shown. Note, the $R_{m}$ value is always less than or equal to that from randomly initialized FCM except for Glass (6 classes). Thirteen data sets have a single extrema for the FCM algorithm. That is, they converge to the same extrema, for all initializations tried here. This is reflected in Table 3 where we have the same values in columns 3 and 4 for the thirteen data sets.

The parameters Number of epochs, $D_{\min }$ and $D_{\max }$ play an important role in determining the quality of the clusters found. By performing manual search, new parameters, which enabled better results by allowing finer search, were found. The values of the new parameters are shown in Table 4 and the results obtained by using these modified parameters are shown 
Table 3: Results for FCM

(Bold entries indicate better $R_{m}$ values than random initialization and italics indicate worse $R_{m}$ values than random initialization)

\begin{tabular}{|c|c|c|c|}
\hline Data Set & $\begin{array}{c}\text { Min } R_{m} \\
\text { found } \\
\text { by Ants } \\
\text { (Std. Dev.) }\end{array}$ & $\begin{array}{c}R_{m} \text { from } \\
\text { FCM, ant } \\
\text { Initialization } \\
\text { (Std. Dev.) }\end{array}$ & $\begin{array}{c}R_{m} \text { from } \\
\text { FCM, random } \\
\text { Initialization } \\
\text { (Std. Dev.) }\end{array}$ \\
\hline British Towns & $1.68(0.0093)$ & $1.60(0.0032)$ & $1.60(0.0033)$ \\
\hline Iris & $5.42(0.0379)$ & $5.23(0)$ & $5.23(0)$ \\
\hline Wine & $33.08(0.4366)$ & $28.71(0)$ & $28.71(0)$ \\
\hline Glass (6 classes) & $11.38(0.4291)$ & $7.293(0.0138)$ & $7.291(0.01)$ \\
\hline Glass (2 classes) & $25.85(0.2246)$ & $24.39(0)$ & $24.39(0)$ \\
\hline $\begin{array}{l}\text { Multiple Sclerosis } \\
\text { (with age) }\end{array}$ & $6.94(0.0208)$ & $6.85(0)$ & $6.85(0)$ \\
\hline $\begin{array}{c}\text { Multiple Sclerosis } \\
\text { (ignoring age) }\end{array}$ & $3.57(0.0213)$ & $3.53(0)$ & $3.53(0)$ \\
\hline MRI & $311.23(3.21)$ & $302.13(1.05)$ & $303.28(2.33)$ \\
\hline Gauss-1 & $6.15(0.15)$ & $5.54(0)$ & $5.54(0)$ \\
\hline Gauss-2 & $4.67(0.15)$ & $4.06(0)$ & $4.06(0)$ \\
\hline Gauss-3 & $4.87(0.15)$ & $4.21(0)$ & $4.21(0)$ \\
\hline Gauss-4 & $2.55(0.17)$ & $1.91(0)$ & $2.74(2.27)$ \\
\hline Gauss-5 & $8.65(0.10)$ & $8.20(0)$ & $8.20(0)$ \\
\hline Gauss500-1 & $4.48(0.06)$ & $4.25(0)$ & $4.25(0)$ \\
\hline Gauss500-2 & $3.63(0.06)$ & $3.36(0)$ & $3.36(0)$ \\
\hline Gauss500-3 & $2.35(0.07)$ & $2.05(0)$ & $2.05(0)$ \\
\hline Gauss500-4 & $1.99(0.06)$ & $1.68(0)$ & $1.78(0.67)$ \\
\hline Gauss500-5 & $1.90(0.07)$ & $1.58(0)$ & $1.58(0)$ \\
\hline
\end{tabular}


in Table 5. Clear improvements were observed for 3 data sets. For the British Towns data set the average value for $R_{m}$ after FCM decreased to 1.5999 from 1.6033, similarly for the Glass (6 classes) data set the average value for $R_{m}$ after FCM decreased to 7.2897 from 7.2937. This average value is better than that obtained from randomly initialized FCM. The average value for $R_{m}$ after FCM for the MRI data set decreased to 301.9198 from 302.1302.

Table 4: New Parameters

\begin{tabular}{|c|r|r|}
\hline Parameter & Old Value & New Value \\
\hline Epochs & 1000 & 2000 \\
\hline$D_{\min }$ & 0.001 & 0.0001 \\
\hline$D_{\max }$ & 0.01 & 0.001 \\
\hline
\end{tabular}

Table 5: Results for FCM obtained from modified parameters (Bold entries indicate better $R_{m}$ values than random initialization)

\begin{tabular}{|c|c|c|c|}
\hline \multirow{2}{*}{ Data Set } & $\begin{array}{c}\text { Min } R_{m} \\
\text { found } \\
\text { by Ants } \\
\text { (Std. Dev.) }\end{array}$ & $\begin{array}{c}R_{m} \text { from } \\
\text { FCM, ant } \\
\text { Initialization } \\
\text { (Std. Dev.) }\end{array}$ & $\begin{array}{c}R_{m} \text { from } \\
\text { FCM, random } \\
\text { Initialization } \\
\text { (Std. Dev.) }\end{array}$ \\
\hline British Towns & $1.6051(0.0015)$ & $\mathbf{1 . 5 9 9 9}(\mathbf{0 . 0 0 3 2})$ & $1.6039(0.0033)$ \\
\hline Glass (6 classes) & $9.2284(0.3417)$ & $\mathbf{7 . 2 8 9 7}(\mathbf{0})$ & $7.2917(0.01)$ \\
\hline MRI & $302.1188(0.0858)$ & $\mathbf{3 0 1 . 9 1 8 9} \mathbf{( 0 . 0 0 0 1 )}$ & $303.2894(2.3354)$ \\
\hline
\end{tabular}

\section{Hard C Means Results}

The ant algorithm was applied with the Hard C Means objective function. The ants find the cluster centers and these centers are used as the initial centers for the Hard C Means algorithm. The parameter values are those shown in Table 1.

From Table 6 we see that the algorithm had lower $J_{1}$ values than randomly initialized HCM for 15 of the 18 data sets tested. Changing the parameter values can improve the results. By performing a search in the parameter space, parameter values that resulted in better partitions were found. Tables 7 and 8 show the variation in the results obtained by changing the number of ants per partition and epochs for the British Towns' and Wine data sets. From the tables we see that as the number of epochs increase, the minimum $R_{1}$ found by the ants decreases, this is to be expected because as the number of epochs increase, the ants 
Table 6: Results for Hard C Means where bold indicates better optimization using the ant based algorithm and italics indicates better optimization using HCM

\begin{tabular}{|c|c|c|c|}
\hline Data Set & $\begin{array}{c}\text { Min } R_{1} \\
\text { found } \\
\text { by Ants } \\
\text { (Std. Dev.) }\end{array}$ & $\begin{array}{c}R_{1} \text { from } \\
\text { HCM, ant } \\
\text { Initialization } \\
\text { (Std. Dev.) }\end{array}$ & $\begin{array}{c}R_{1} \text { from } \\
\text { HCM, random } \\
\text { Initialization } \\
\text { (Std. Dev.) }\end{array}$ \\
\hline British Towns & $5.5202(.05545)$ & $3.6260(0.4093)$ & $3.4339(0.3759)$ \\
\hline Iris & $7.0055(0.0025)$ & $6.9981(0)$ & $8.2516(2.0701)$ \\
\hline Wine & $52.8098(5.9252)$ & $50.4573(4.4826)$ & 48.9792 (0.0135) \\
\hline Glass (6 classes) & $28.1317(1.3873)$ & $24.3770(1.9901)$ & 21.1165 (2.3599) \\
\hline Glass (2 classes) & $34.2488(0.0537)$ & $34.1352(0.0050)$ & $36.9132(6.5047)$ \\
\hline $\begin{array}{l}\text { Multiple Sclerosis } \\
\text { (with age) }\end{array}$ & $10.22010 .1260)$ & $10.2016(0.1310)$ & $10.3548(0.5711)$ \\
\hline $\begin{array}{l}\text { Multiple Sclerosis } \\
\text { (ignoring age) }\end{array}$ & $4.6406(0.0011)$ & $4.6381(0)$ & $4.7759(0.8145)$ \\
\hline MRI & $433.2752(0.2880)$ & $432.7499(0)$ & $452.384(37.7701)$ \\
\hline Gauss-1 & $7.1391(0.1559)$ & $6.4856(0)$ & $11.0962(8.4406)$ \\
\hline Gauss-2 & $5.1055(0.1442)$ & $4.4725(0)$ & $11.0645(11.3973)$ \\
\hline Gauss-3 & $5.2625(0.1510)$ & $4.6624(0)$ & $12.7167(14.1517)$ \\
\hline Gauss-4 & $2.7273(0.1844)$ & $2.0386(0)$ & $12.4133(15.5424)$ \\
\hline Gauss-5 & $11.6107(0.1521)$ & $10.9422(0)$ & $11.9558(5.0158)$ \\
\hline Gauss500-1 & $5.8333(0.0814)$ & $5.4921(0)$ & $6.0718(2.5999)$ \\
\hline Gauss500-2 & $4.3405(0.0908)$ & $4.0029(0)$ & $7.7512(6.5174)$ \\
\hline Gauss500-3 & $2.6558(0.0834)$ & $2.3140(0)$ & $6.3784(5.7135)$ \\
\hline Gauss500-4 & $2.1678(0.0972)$ & $1.8465(0)$ & $7.3442(4.6114)$ \\
\hline Gauss500-5 & $2.0758(0.0865)$ & $1.7314(0)$ & $7.0953(5.8005)$ \\
\hline
\end{tabular}


get more time to refine the centroids found. Also as the number of ants increase, lower $R_{1}$ values are found. Table 9 shows the results obtained for different MRI slices, the parameter values used for the MRI data set are the originals from Table 4 and ants equivalent to 50 partitions were used. All the partitions have lower $R_{1}$ values than HCM.

Table 7: Results for the British Towns data set with HCM

\begin{tabular}{|r|c|c|c|c|}
\hline Partitions & Epochs & $\begin{array}{c}\text { Min } R_{1} \\
\text { found } \\
\text { by Ants } \\
\text { (Std. Dev.) }\end{array}$ & $\begin{array}{c}R_{1} \text { from } \\
\text { HCM, ant } \\
\text { Initialization } \\
\text { (Std. Dev.) }\end{array}$ & $\begin{array}{c}R_{1} \text { from } \\
\text { HCM, random } \\
\text { Initialization } \\
\text { (Std. Dev.) }\end{array}$ \\
\hline 50 & 2000 & $5.3658(0.4989)$ & 3.5812 (0.2869) & \multirow{2}{*}{3.4339 (0.3759) } \\
\hline 50 & 4000 & $4.5048(0.3031)$ & 3.5701 (0.3010) & \\
\hline 75 & 2000 & $5.1691(0.4406)$ & 3.6134 (0.3561) & \\
\hline 100 & 2000 & $3.0835(0.0229)$ & $\mathbf{3 . 0 6 6 1 ( \mathbf { 0 . 0 2 3 1 } )}$ & \\
\hline
\end{tabular}

Table 8: Results for the Wine data set with HCM

\begin{tabular}{|c|c|c|c|c|}
\hline Partitions & Epochs & $\begin{array}{c}\text { Min } R_{1} \\
\text { found } \\
\text { by Ants } \\
\text { (Std. Dev.) }\end{array}$ & $\begin{array}{c}R_{1} \text { from } \\
\text { HCM, ant } \\
\text { Initialization } \\
\text { (Std. Dev.) }\end{array}$ & $\begin{array}{c}R_{1} \text { from } \\
\text { HCM, random } \\
\text { Initialization } \\
\text { (Std. Dev.) }\end{array}$ \\
\hline 50 & 2000 & $52.8003(5.6307)$ & $49.2405(1.8660)$ & \multirow{6}{*}{$48.9792(0.0143)$} \\
\hline 50 & 4000 & $51.0631(4.7890)$ & $49.2604(2.6368)$ & \\
\hline 75 & 2000 & $50.1879(2.8663)$ & $49.2604(2.0259)$ & \\
\hline 75 & 3000 & $49.9230(2.8418)$ & $48.9748(0.0186)$ & \\
\hline 100 & 2000 & $49.6415(0.3717)$ & $48.9716(0.0152)$ & \\
\hline 100 & 4000 & $49.2076(0.0517)$ & $48.9697(0.0143)$ & \\
\hline
\end{tabular}

\section{Execution Time}

The variation of the minimum $R_{m}$ (using FCM) found by the ants by changing the number of ants to allow for more partitions for MRI slice \# 35 is shown in Table 10. As the number of ants increases the minimum $R_{m}$ found decreases, but at the cost of increased execution time. As the number of ants increase, more search space is explored and we got lower $R_{m}$ values. 
Table 9: Results for the MRI data set with HCM

\begin{tabular}{|c|c|c|c|}
\hline $\begin{array}{c}\text { Slice } \\
\#\end{array}$ & $\begin{array}{c}\text { Min } R_{1} \\
\text { found } \\
\text { by Ants } \\
\text { (Std. Dev.) }\end{array}$ & $\begin{array}{c}R_{1} \text { from } \\
\text { HCM, ant } \\
\text { Initialization } \\
\text { (Std. Dev.) }\end{array}$ & $\begin{array}{c}R_{1} \text { from } \\
\text { HCM, random } \\
\text { Initialization } \\
\text { (Std. Dev.) }\end{array}$ \\
\hline 20 & $853.7991(1.1036)$ & $\mathbf{8 5 1 . 8 3 4 2}(\mathbf{0 . 0 0 1 2})$ & $882.5732(65.3882)$ \\
\hline 35 & $919.8082(22.1066)$ & $\mathbf{9 1 7 . 6 6 3 6}(\mathbf{2 2 . 3 6 7 6 )}$ & $927.6961(33.5836)$ \\
\hline 45 & $839.1622(0.5126)$ & $\mathbf{8 3 8 . 0 1 7 5}(\mathbf{0})$ & $851.3756(28.8006)$ \\
\hline 46 & $842.5583(0.5615)$ & $\mathbf{8 4 1 . 3 4 1 4} \mathbf{( 0 )}$ & $847.0730(15.6787)$ \\
\hline 47 & $796.8415(0.5191)$ & $\mathbf{7 9 5 . 6 0 5 7}(\mathbf{0 . 4 4 3 9})$ & $834.1028(73.2008)$ \\
\hline
\end{tabular}

Table 10: Variation of $R_{m}$ with the number of ants for slice \# 35 of MRI data set

\begin{tabular}{|c|c|c|c|}
\hline Partitions & $\begin{array}{c}\text { Min } R_{m} \\
\text { found } \\
\text { by Ants } \\
\text { (Std. Dev.) }\end{array}$ & $\begin{array}{c}R_{m} \text { from } \\
\text { HCM, ant } \\
\text { Initialization } \\
\text { (Std. Dev.) }\end{array}$ & $\begin{array}{c}R_{m} \text { from } \\
\text { HCM, random } \\
\text { Initialization } \\
\text { (Std. Dev.) }\end{array}$ \\
\hline 50 & $919.8082(22.1066)$ & $917.6636(22.3676)$ & \multirow{3}{*}{$927.6961(33.5836)$} \\
\hline 75 & $912.0365(16.7062)$ & $909.9324(16.9365)$ & \\
\hline 100 & $910.0054(14.3460)$ & $907.9996(14.6430)$ & \\
\hline
\end{tabular}


The execution time for the British Towns and the MRI data set is shown in Table 11. These two data sets were chosen because the British Towns' data set is the smallest (in terms of number of examples) data set and the MRI data set is the largest data set used in the study. The values are an average from 20000 epochs and 5 experiments for the British Towns' data set and from 6000 epochs and 3 experiments for the MRI data set. One experiment consists of the ant stage and the following FCM or the Hard C Means stage. The time required for one epoch for the British towns data set was more than that for the MRI data set as there are more classes, and hence more ants, in the British towns data set. The time required for the entire experiment was more for the MRI data set as there are more examples in the MRI data set. The time required for the FCM algorithm is also shown in Table 11. From Table 11 we see that, for the British Towns' data set, approximately 9100 random FCM initializations can be performed in the time required for one ant experiment. Similarly approximately 225 random FCM initializations can be performed for the MRI data set.

We performed $11300(\approx 225 \times 50)$ random initializations for the MRI data set and averaged the best $50 R_{m}$ values. The average $R_{m}$ value was 301.9189 and the average $R_{m}$ value found by ants from 50 random initializations was 301.9189. Similarly for the British Towns', data set the average of best $50 R_{m}$ values over 20000 iterations was 1.5999 and the average $R_{m}$ value found by ants, from 50 random initializations, was 1.5999 .

The experiments were performed on an Intel Pentium $42.53 \mathrm{GHz}$ processor with $512 \mathrm{~KB}$ cache and $2 \mathrm{~GB}$ of main memory.

Table 11: Execution Time

\begin{tabular}{|c|r|r|r|r|}
\hline \multirow{2}{*}{ Data Set } & Partitions & $\begin{array}{c}\text { Time for } \\
\text { one Epoch } \\
\text { (milliseconds) }\end{array}$ & $\begin{array}{r}\text { Time for } \\
\text { one Experiment } \\
\text { (seconds) }\end{array}$ & $\begin{array}{c}\text { Time for } \\
\text { FCM } \\
\text { (seconds) }\end{array}$ \\
\hline \multirow{3}{*}{ British Towns } & 50 & 14.9645 & 97 & \multirow{2}{*}{0.01318} \\
\cline { 2 - 4 } & 75 & 22.2210 & 108.8380 & 120.896 \\
\hline \multirow{2}{*}{ MRI } & 100 & 29.7060 & 540.72 & \multirow{2}{*}{2.3929} \\
\cline { 2 - 4 } & 50 & 6.7333 & 811.0133 & \\
\hline
\end{tabular}

\section{Discussion}

The ants move the cluster centers in feature space to find a good partition for the data. There are less controlling parameters than the previous ant based clustering algorithms [5]. 
The previous ant clustering algorithms typically group the objects on a two-dimensional grid.

Results from 18 data sets show the superiority of our algorithm over the randomly initialized FCM and HCM algorithms. For comparison purposes, Tables 12, 13 and 14 show the frequency of occurrence of different extrema for the ant initialized FCM and HCM algorithms and the randomly initialized FCM and HCM algorithms. The ant initialized FCM algorithm always finds better extrema for the MRI data set and for the British Towns' data set the ant initialized algorithm finds the better extrema 49 out of 50 times. The ant initialized HCM algorithm always finds better extrema for the Iris data set and for the Glass (2 class) data set a majority of the time. For the different MRI slices, the ant initialized HCM algorithm finds a better extrema most of the time. In [22], a Genetic programming approach was used to optimize the clustering criteria, the genetic approach for Hard C Means, found better extrema $64 \%$ of the time for the Iris data set. The ant initialized HCM finds better extrema all the time.

The number of ants is an important parameter of the algorithm. This number only increases when more partitions are searched for at the same time; as ants are (currently) added in $c \times s$ increments. The quality of the final partition improves with an increase of ants, but the improvement comes at the expense of increased execution time. Future work might focus on automatically finding the minimal number of ants needed.

It would be possible, and perhaps quite helpful, to do a couple of iterations of a standard iterative clustering algorithm to refine the obtained partition after every epoch. This might well result in equivalent partitions in perhaps the same or less time.

Table 12: Frequency of different extrema from FCM, for British Towns and MRI data set

\begin{tabular}{|c|r|r|r|}
\hline Data Set & Extrema & $\begin{array}{c}\text { Frequency } \\
\text { FCM, ant } \\
\text { Initialization }\end{array}$ & $\begin{array}{c}\text { Frequency } \\
\text { FCM, random } \\
\text { Initialization }\end{array}$ \\
\hline \multirow{2}{*}{$\begin{array}{c}\text { British } \\
\text { Towns }\end{array}$} & 1.5999 & 49 & 16 \\
\cline { 2 - 4 } & 1.6037 & 1 & 18 \\
\hline \multirow{2}{*}{ MRI } & 301.9195 & 0 & 16 \\
\cline { 2 - 4 } & 307.1898 & 50 & 37 \\
\hline
\end{tabular}


Table 13: Frequency of different extrema from HCM, for Glass (2 class) and Iris data set

\begin{tabular}{|c|r|r|r|}
\hline Data Set & Extrema & $\begin{array}{c}\text { Frequency } \\
\text { HCM, ant } \\
\text { Initialization }\end{array}$ & $\begin{array}{c}\text { Frequency } \\
\text { HCM, random } \\
\text { Initialization }\end{array}$ \\
\hline \multirow{4}{*}{$\begin{array}{c}\text { Glass } \\
(2 \text { class })\end{array}$} & 34.1320 & 19 & 3 \\
\cline { 2 - 4 } & 34.1343 & 11 & 19 \\
\cline { 2 - 4 } & 34.1372 & 19 & 15 \\
\hline \multirow{4}{*}{ Iris } & 34.1658 & 1 & 5 \\
\cline { 2 - 4 } & 6.9981 & 50 & 23 \\
\cline { 2 - 4 } & 10.1386 & 0 & 14 \\
\cline { 2 - 4 } & 12.1437 & 0 & 5 \\
\hline
\end{tabular}

Table 14: Frequency of different extrema from HCM, MRI data set

\begin{tabular}{|c|l|r|r|}
\hline $\begin{array}{c}\text { Slice } \\
\text { \# }\end{array}$ & Extrema & $\begin{array}{c}\text { Frequency } \\
\text { HCM, ant } \\
\text { Initialization }\end{array}$ & $\begin{array}{c}\text { Frequency } \\
\text { HCM, random } \\
\text { Initialization }\end{array}$ \\
\hline \multirow{2}{*}{20} & 841.3414 & 50 & 44 \\
\cline { 2 - 4 } & 889.1043 & 0 & 6 \\
\hline \multirow{4}{*}{35} & 930.1677 & 45 & 30 \\
\cline { 2 - 4 } & 951.4871 & 5 & 15 \\
\cline { 2 - 4 } & 1003.492 & 0 & 5 \\
\hline \multirow{3}{*}{45} & 838.0175 & 50 & 41 \\
\cline { 2 - 4 } & 912.2289 & 0 & 9 \\
\hline \multirow{3}{*}{46} & 841.3414 & 50 & 45 \\
\cline { 2 - 4 } & 889.1043 & 0 & 5 \\
\hline \multirow{3}{*}{47} & 795.3043 & 35 & 27 \\
\cline { 2 - 4 } & 796.2459 & 15 & 13 \\
\cline { 2 - 4 } & 970.5483 & 0 & 10 \\
\hline
\end{tabular}




\subsection{Finding the number of clusters}

The clustering approach described here requires knowledge of the number of clusters. It is possible to use cluster validity metrics on the resulting partitions created with different numbers of clusters to determine the right number of clusters [3, 30]. Alternatively, one might replace the function $R_{m}$ or $R_{1}$ being optimized by the swarm of ants with a partition validity metric. Perhaps the ants would place clusters that were not needed in locations where few, if any, examples would be closer to them than others.

We have explored this possibility using the Xie-Beni partition validity metric which is used for fuzzy partitions. It can be described as in [31]:

$$
X B(\beta, X)=\frac{R_{m}(\beta, X)}{n\left(\min _{i \neq j}\left\{\left\|\beta_{i}-\beta_{j}\right\|^{2}\right\}\right)}
$$

It is clearly tied to the FCM functional with a strong preference for keeping the smallest distance between any two cluster centroids as large as possible. The smallest $X B(\beta, X)$ is considered to be the best.

So, we just replace $R_{m}$ with XB in Algorithm 1 and begin with an overestimate of the number of clusters. Generally, we expect it will be possible to guess a number greater than the likely number of clusters (and less than the number of examples).

\subsubsection{Choosing the number of clusters experiments}

Experiments were done with the Wine and Iris data sets discussed earlier. Also, an artificial data set with 2 attributes, 5 classes and 1000 examples was generated using a Gaussian distribution. It is shown in Figure 5. The classes are slightly unequally sized [8] (248, 132, 217, 192 and 211 respectively).

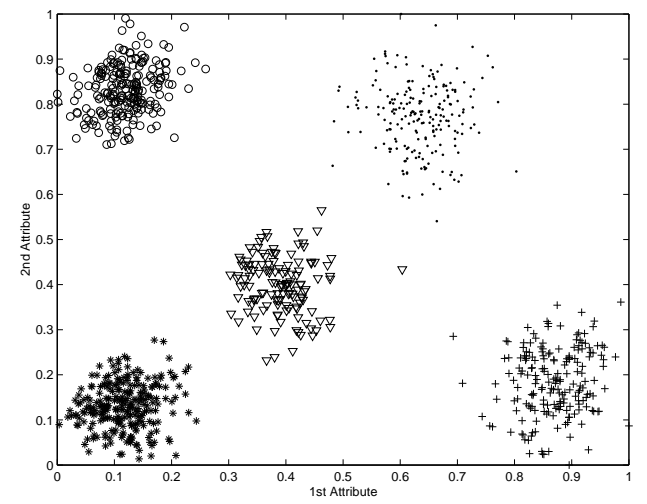

Figure 5: Gauss-1 Dataset (Normalized) 
The default parameters were used. Each experiment was done with the known number of clusters or more. For the Iris data set, we also tried two classes because of the fact that in feature space an argument can be made for this number of classes.

We will look at the results from the Iris data set first. When we tried to cluster into three classes; a partition with 50 examples from class 1 and 100 examples from class 2/class 3 was found 10 of 30 times. The rest of the time a cluster with one example was found four times and in the other experiments the cluster with class 1 had a few examples from another class. So, the results seem to clearly indicate that there are two classes.

However, a repeatable method that could objectively determine how many classes existed was necessary. We used a threshold on the number of examples in a cluster. The FCM functional has a bias towards producing approximately equal size clusters. It is not the right functional to use for widely different sized clusters. Hence, we used a threshold which was the percent of examples assuming each cluster was the same size. If a cluster had less than the threshold, we interpreted that to mean there was no cluster and the cluster should be merged with the one closest in feature space. We did not, in these experiments, try to merge the clusters. The equation is

$$
T=\frac{n}{c} * P,
$$

where $n$ is the number of examples, $c$ is the number of clusters searched for and $P$ is the percentage of the average cluster size. Any percentage 2 or greater will lead to the conclusion that there are only 2 clusters in the Iris data when we search for 3 . Results are summarized for different $c$ in Table 15.

Table 15: Number of clusters searched for and average number found for the Iris data with the minimum $\mathrm{P}$ over 30 trials

\begin{tabular}{|c|c|c|}
\hline $\begin{array}{c}\text { Clusters } \\
\text { searched }\end{array}$ & $\begin{array}{c}\text { Ave. clusters } \\
\text { found }\end{array}$ & $\mathrm{P}$ \\
\hline 3 & 2 & 0.02 \\
4 & 2 & 0.3 \\
5 & 2 & 0.9 \\
6 & 2.5 & 0.9 \\
\hline
\end{tabular}

Searching for four clusters in the Iris data, a partition with 50 examples from class 1 and the other two classes perfectly mixed occurred three times. There was always one empty cluster and the largest cluster size was 9 in the case that three clusters were found. So, any threshold above 30 percent will provide the conclusion that there are only two clusters.

With five clusters there were typically two or three empty clusters and the "perfect" partition into two clusters occurs twice. If a percentage of 90 or above is used the conclusion will be two clusters exist. This search space is significantly larger and no more epochs were 
utilized, so it appears the result is a strong one. We also tried six clusters where there were typically two or three empty clusters. In this case, with a percentage of 90 or above the average number of classes was 2.5. There were a number of cases in which the linearly separable class would get discovered as one cluster and the other two classes would be split into two (maybe $67 / 33$ or $74 / 26$ for example). Again, in this large search space this seems to be a very reasonable result. One would probably not guess double (or triple!) the number of actual classes.

In order to evaluate whether a more complete search might result in the discovery of 2 clusters more often when we initially searched for 6 , we changed the number of epochs to 4000 and the number of iterations per epochs to 25. This causes the ant to move less during epochs and have more opportunities (epochs) to find good partitions. With these parameters and a percentage of 90 , just 2 clusters were found for all thirty trials. The examples in the linearly separable class were assigned, by themselves, to one cluster nine times.

Finally, we report the results when searching for only 2 clusters. In this case, there were always two clusters found (for $P<0.65$ ). In 14/30 trials a partition with the linearly separable class and the other two classes mixed was found. In the other experiments a few examples were assigned with the linearly separable class making its size between 51 and 54 . So, a very reasonable partition was obtained when searching for two classes.

For the artificial data we did experiments with 5, 6, 7, 8 and 9 clusters. Results are summarized for different $c$ in Table 16. The ant based clustering always found five clusters when it started with five. In fact, it found the exact original partition 15 times. When it was incorrect, it had some small confusion between class two and class five. A typical partition that did not match the original was: $(248,133,217,192,210)$ in which one example had switched between class 2 and class 5 . This seems to be a pretty reasonable clustering result given the larger search space of the ants.

Table 16: Number of clusters searched for and average number found for the Artificial data with the minimum $\mathrm{P}$ over 30 trials.

\begin{tabular}{|c|c|c|}
\hline $\begin{array}{c}\text { Clusters } \\
\text { searched }\end{array}$ & $\begin{array}{c}\text { Ave. clusters } \\
\text { found }\end{array}$ & $\mathrm{P}$ \\
\hline 6 & 5 & 0.3 \\
7 & 5.033 & 0.3 \\
8 & 5 & 0.75 \\
9 & 5 & 0.8 \\
\hline
\end{tabular}

When it searched for six classes, it always found five for a $P \geq 0.3$ or greater. The sixth cluster typically had between 0 and two examples assigned to it. When searching for seven 
classes, it found five classes 29 times for $P \geq 0.3$. One time it found six classes. In that case there was an empty cluster and then class 4 was split into two clusters.

For eight classes, exactly five were found for a $P=0.75$. Making it larger would occasionally cause 4 to be found when Cluster 5 was split exactly into 2 chunks. For nine classes, five classes were always found for a percentage of 80 up to about 90. There might be two or three empty clusters. The other non-clusters were very lightly populated with less than 15 examples closest to their centroid in the usual case. As the percentage got too high it would cause a class split into two, to occasionally be missed resulting in four clusters. For example, with $P=1, T=111.11$ class 4 was split into two clusters with 107 and 86 examples in each, respectively.

\section{Conclusions}

We discussed a swarm inspired optimization algorithm to partition or create clusters of data. We described it using the ant paradigm. The approach is to have a coordinated group of ants position cluster centroids in feature space.

The algorithm was evaluated with a soft clustering formulation utilizing the fuzzy c-means objective function and a hard clustering formulation utilizing the hard c-means objective function.

The presented clustering approach seems clearly advantageous for the data sets where it is expected there will be lots of local extrema. The cluster discovery aspect of the algorithm provides the advantage of obtaining a partition at the same time it indicates the number of clusters. That partition can be further optimized or accepted as is. This is in contrast to some other schemes which require partitions to be created with different numbers of clusters and then evaluated.

The results are generally a better optimized partition (objective function) than obtained with FCM/HCM. One needs a large number of random initializations to be competitive in terms of skipping some of the poor local extrema which was done with the ant-based algorithm. It has provided better final partitions on average than a previously introduced evolutionary computation clustering approach for several data sets. Random initializations have been shown to be the best approach for the c-means family [26] and the ant clustering algorithm results in generally better partitions than a single random initialization. A parallel version of the ants algorithm could operate much faster than the current sequential implementation, thereby making it a clear choice for minimizing the chance of finding a poor extrema when doing c-means clustering. This algorithm should scale better for large numbers of examples than grid-based ant clustering algorithms. 


\section{References}

[1] A. Baraldi and P. Blonda, "A survey of fuzzy clustering algorithms for pattern recognition. i," IEEE Transactions on Systems, Man and Cybernetics, Part B, vol. 29, no. 6, pp. 778-785, 1999.

[2] A. K. Jain and R. C. Dubes, Algorithms for Clustering Data. Prentice Hall, 1988.

[3] J. Bezdek, J. Keller, R. Krishnapuram, and N. Pal, Fuzzy Models and Algorithms for Pattern Recognition and Image Processing. Boston, MA: Kluwer, 1999.

[4] E. Bonabeau, M. Dorigo, and G. Theraulaz, Swarm Intellignece From Natural to Artificial Systems. New York, NewYork: Oxford University Press, 1999.

[5] P. M. Kanade and L. O. Hall, "Fuzzy ants as a clustering concept," North American Fuzzy Information Processing Society, NAFIPS 2003, 22nd International Conference of the, pp. 227-232, 2003.

[6] F. Höppner, F. Klawonn, R. Kruse, and T. Runkler, Fuzzy Cluster Analysis. West Sussex, England: John Wiley, 1999.

[7] J. C. Bezdek, "Pattern recognition with fuzzy ojective functions algorithms," Plenum press, 1981.

[8] P. Kanade, "Fuzzy ants as a clustering concept," Master's thesis, University of South Florida, Tampa, FL, 2004.

[9] A. Ultsch, "Strategies for an artificial life system to cluster high dimensional data," in Abstracting and Synthesizing the Principles of Living Systems, GWAL-6, U. Brggemann, H. Schaub, and F. Detje, Eds., 2004, pp. 128-137.

[10] W. Lai, K. Hoe, T. Tai, and M. Seah, "Classifying english web pages with "smart" ant-like agents soft computing," in Multimedia Biomedicine, Image Processing and Financial Engineering Vol.13, 2002, pp. 411-416.

[11] N. Monmarché, M. Slimane, and G. Venturini, "On improving clustering in numerical databases with artificial ants," in 5th European Conference on Artificial Life (ECAL'99), Lecture Notes in Artificial Intelligence, D. Floreano, J. Nicoud, and F. Mondala, Eds., vol. 1674. Lausanne, Switzerland: Springer-Verlag, Sep 1999, pp. 626-635. 
[12] — - "Antclass: discovery of clusters in numeric data by an hybridization of an ant colony with the kmeans algorithm," Laboratoire d'Informatique de l'Universite de, Jan 1999.

[13] J. Handl and B. Meyer, "Improved ant-based clustering and sorting in a document retrieval interface," in Proceedings of the Seventh International Conference on Parallel Problem Solving from Nature (PPSN VII), vol. LNCS 2439, 2002, pp. 913-923.

[14] Y. Yang and M. Kamel, "Clustering ensemble using swarm intelligence," in Proceedings of the 2003 IEEE Swarm Intelligence Symposium. SIS'03, 2003, pp. 65-71.

[15] E. Lumer and B. Faieta, "Diversity and adaptation in populations of clustering ants," in Proceedings of the Third International Conference on Simulation of Adaptive Behavior: from Animals to Animats. MIT Press, 1994, pp. 499-508.

[16] J. Handl, J. Knowles, and M. Dorigo, "On the performance of ant-based clustering. design and application of hybrid intelligent systems," in Frontiers in Artificial intelligence and Applications 104, 2003, pp. 204-213.

[17] — "Strategies for the increased robustness of ant-based clustering," in SelfOrganising Applications: Issues, challenges and trends, vol. LNCS 2977, 2003, pp. 90-104.

[18] S. Ouadfel and M. Batouche, "Unsupervised image segmentation using a colony of cooperating ants," in Biologically Motivated Computer Vision, Second International Workshop, BMCV 2002, vol. Lecture Notes in Computer Science Vol.2525, 2002, pp. 109-116.

[19] A. Strehl and J. Ghosh, "Clusters ensembles- a knowledge reuse framework for combining multiple partitions," Journal of Machine learning Research, vol. 3, pp. 583-617, 2002.

[20] H. Azzag, N. Monmarché, M. Slimane, G. Venturini, and C. Guinot, "Anttree : a new model for clustering with artificial ants," in IEEE Congress on Evolutionary Computation, 2003.

[21] N. Labroche, N. Monmarche, and G. Venturini, "A new clustering algorithm based on the chemical recognition system of ants," in Proceedings of the European Conference on Artificial Intelligence, 2002, pp. 345-349. 
[22] L. O. Hall, I. B. Özyurt, and J. C. Bezdek, "Clustering with a genetically optimized approach," IEEE Trans. on Evolutionary Computation, vol. 3, no. 2, pp. 103-112, 1999.

[23] R. J. Hathway and J. C. Bezdek, "Optimization of clustering criteria by reformulation," IEEE Transactions on Fuzzy Systems, vol. 3, no. 2, pp. 241-245, May 1995.

[24] N. Belacel, P. Hansen, and N. X. Mladenovic, "Fuzzy j-means," Pattern recognition, vol. 35, pp. 2193-2200, 2002.

[25] S. Khan and A. Ahmad, "Cluster center initialization algorithm for k-means clustering," Pattern recognition Letters, vol. 25, pp. 1293-1302, 2004.

[26] J. Pena, J. Lozano, and P. Larranaga, "An empirical comparison of four initialization methods for the k-means algorithm," Pattern recognition Letters, vol. 20, pp. 1027-1040, 1999.

[27] A. Inselberg, "The plane with parallel coordinates," Special Issue on Computational Geometry, The Visual Computer, no. 1, pp. 69-97, 1985.

[28] M. Berthold and L. Hall, "Visualizing fuzzy points in parallel coordinates," IEEE Transactions on Fuzzy Systems, vol. 11, no. 3, pp. 369-374, June 2003.

[29] R. Duda and P. Hart, Pattern Classification and Scene Analysis. New York: John Wiley and Sons, 1973.

[30] N. Pal and J. Bezdek, "On cluster validity for the fuzzy c-means model," IEEE Transactions on Fuzzy Systems, vol. 3, no. 3, pp. 370-379, 1995.

[31] X. Xie and G. Beni, "Validity measure for fuzzy clustering," IEEE Transactions on Pattern Analysis and Machine Intelligence, vol. 3, no. 8, pp. 841-846, 1991. 
Appendix A

Table 17: Parameters used to generate the Gaussian data sets

\begin{tabular}{|c|c|c|c|c|}
\hline Data Set & \multicolumn{2}{|c|}{ Mean } & \multicolumn{2}{|c|}{ Std. Dev } \\
\hline \multirow{5}{*}{ Gauss-1 } & 2 & 2 & 1 & \\
\hline & 8 & 8 & 1 & 1 \\
\hline & 2 & 16 & 1 & 1 \\
\hline & 19 & 3 & 1 & 1 \\
\hline & 14 & 14 & 1 & 1 \\
\hline \multirow{5}{*}{ Gauss-2 } & 2 & 2 & 1 & 1 \\
\hline & 10 & 10 & 1 & 1 \\
\hline & 3 & 20 & 1 & 1 \\
\hline & 22 & 5 & 2 & 2 \\
\hline & 18 & 18 & 1 & 1 \\
\hline \multirow{5}{*}{ Gauss-3 } & 2 & 4 & 1 & 1 \\
\hline & 11 & 11 & 1 & 1 \\
\hline & 5 & 20 & 1 & 1 \\
\hline & 22 & 5 & 2 & 2 \\
\hline & 20 & 20 & 1 & 1 \\
\hline \multirow{5}{*}{ Gauss-4 } & 3 & 3 & 1 & 1 \\
\hline & 11 & 11 & 1 & 1 \\
\hline & 10 & 30 & 1 & 1 \\
\hline & 32 & 5 & 1 & 1 \\
\hline & 20 & 33 & 1 & 1 \\
\hline \multirow{5}{*}{ Gauss-5 } & 2 & 2 & 1 & 1 \\
\hline & 6 & 6 & 1 & 1 \\
\hline & 2 & 10 & 1 & 1 \\
\hline & 12 & 4 & 2 & 2 \\
\hline & 11 & 11 & 1 & 1 \\
\hline \multirow{5}{*}{ Gauss-500-1 } & 4 & 4 & 1 & 1 \\
\hline & 8 & 8 & 1 & 1 \\
\hline & 3 & 12 & 1 & 1 \\
\hline & 11 & 4 & 2 & 2 \\
\hline & 12 & 12 & 1 & 1 \\
\hline \multirow{5}{*}{ Gauss-500-2 } & 4 & 4 & 1 & 1 \\
\hline & 7 & 10 & 1 & 1 \\
\hline & 4 & 16 & 1 & 1 \\
\hline & 13 & 4 & 1 & 1 \\
\hline & 14 & 14 & 1 & 1 \\
\hline
\end{tabular}


Table 18: Parameters used to generate the Gaussian data sets

\begin{tabular}{|c|r|r|r|r|}
\hline Data Set & \multicolumn{2}{|c|}{ Mean } & \multicolumn{2}{l|}{ Std. Dev } \\
\hline \multirow{4}{*}{ Gauss-500-3 } & 4 & 6 & 1 & 1 \\
\cline { 2 - 5 } & 12 & 15 & 1 & 1 \\
\cline { 2 - 5 } & 4 & 21 & 1 & 1 \\
\cline { 2 - 5 } & 13 & 4 & 1 & 1 \\
\cline { 2 - 5 } Gauss-500-4 & 18 & 14 & 1 & 1 \\
\hline & 4 & 6 & 1 & 1 \\
\cline { 2 - 5 } & 12 & 16 & 1 & 1 \\
\cline { 2 - 5 } & 4 & 21 & 1 & 1 \\
\cline { 2 - 5 } & 13 & 4 & 2 & 2 \\
\cline { 2 - 5 } & 24 & 14 & 1 & 1 \\
\hline \multirow{5}{*}{ Gauss-500-5 } & 6 & 8 & 1 & 1 \\
\cline { 2 - 5 } & 15 & 15 & 1 & 1 \\
\cline { 2 - 5 } & 9 & 21 & 1 & 1 \\
\cline { 2 - 5 } & 16 & 4 & 2 & 2 \\
\cline { 2 - 5 } & 28 & 18 & 1 & 1 \\
\hline
\end{tabular}




\section{List of Figures}

1 Fuzzy C Means Algorithm . . . . . . . . . . . . . . . . . 5

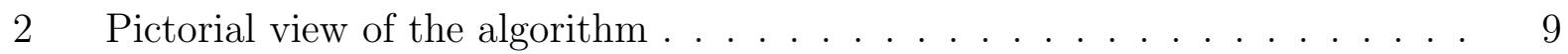

3 Fuzzy ant clustering with centroids algorithm . . . . . . . . . . 10

4 Iris Data Set (Normalized)- First 2 Principal Components . . . . . . . . . . 12

5 Gauss-1 Dataset (Normalized) . . . . . . . . . . . . . . . . . . . . . 21

\section{List of Tables}

1 Parameter Values . . . . . . . . . . . . . . . . . . . . . . . . . . . 11

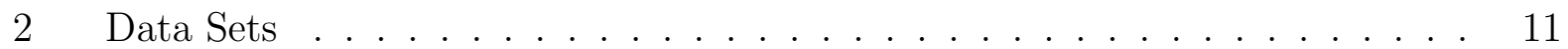

3 Results for FCM . . . . . . . . . . . . . . . . . . . . . 13

4 New Parameters. . . . . . . . . . . . . . . . . . . . . . . . . . . 14

5 Results for FCM obtained from modified parameters . . . . . . . . . . . 14

6 Results for Hard C Means . . . . . . . . . . . . . . . . . . . . . . 15

7 Results for the British Towns data set f . . . . . . . . . . . . 16

8 Results for the Wine data set . . . . . . . . . . . . . . . 16

9 Results for the MRI data set . . . . . . . . . . . . . . . . . . . 17

10 Variation of $R_{m}$ with the number of ants for slice \# 35 of MRI data set . . . 17

11 Execution Time . . . . . . . . . . . . . . . . . . . . 18

12 Frequency of different extrema from FCM, for British Towns and MRI data set 19

13 Frequency of different extrema from HCM, for Glass (2 class) and Iris data set 20

14 Frequency of different extrema from HCM, MRI data set . . . . . . . . . . 20

15 Number of clusters searched for and average number found for the Iris data with the minimum $\mathrm{P}$ over 30 trials. . . . . . . . . . . . . . . . . 22

16 Number of clusters searched for and average number found for the Artificial data with the minimum $\mathrm{P}$ over 30 trials. . . . . . . . . . . . . . . 23

17 Parameters used to generate the Gaussian data sets . . . . . . . . . . . 28

18 Parameters used to generate the Gaussian data sets continued . . . . . . . . 29 